\title{
XIII. A continuous and alternating current magnetic curve tracer
}

John Ely Moore M.E. E.E.

To cite this article: John Ely Moore M.E. E.E. (1896) XIII. A continuous and alternating current magnetic curve tracer, Philosophical Magazine Series 5, 41:249, 106-117, DOI: $10.1080 / 14786449608620820$

To link to this article: http://dx.doi.org/10.1080/14786449608620820

曲 Published online: 08 May 2009.

Submit your article to this journal $[\pi$

Џ Article views: 2

Q View related articles $\asymp$ 
inconsistent with the existence of a current of about a tenth or a twentieth of that amount; but on account both of the smallness of the results and of the discrepancy between the values obtained for 1891 by two methods, we cannot assert that such a current actually exists. The calculations taken by themselves do not disprove the hypothesis that electric currents traverse the earth's surface, as we cannot argue from the condition of a small portion of the globe to that of the whole. The most that can be said is that no evidence in favour of the existence of vertical currents can be drawn from one district, which has been very minutely surveyed.

P.S.-No reference to Dr. Schmidt's original paper was given in the short notice published in the Report of the British Association. I had therefore supposed that the latter was a preliminary note. Professor Schuster has, however, recently shown to me Dr. Schmidt's complete investigation, and he has kindly calculated the current-density at latitudes $50^{\circ}$ and $55^{\circ}$ on the prime meridian from formulæ given by Dr. Schmidt. The result is upward currents and 0.20 to 0.15 ampere per square kilometre at lat. $50^{\circ}$ and $55^{\circ}$ respectively. The mean of these two numbers, viz. $0 \cdot 175$, is nearly equal to Schmidt's mean for the whole earth $(0 \cdot 17)$. It is opposite in direction to and very much greater in magnitude than any vertical current the existence of which is compatible with the results of the Magnetic Survey.

XIII. A Continuous and Alternating Current Magnetic Curve Tracer. By JoHN Ely Moore, M.E., E.E.*

\$1. $\mathrm{F}$ the various methods that have been pruposed and used for measuring the magnetic quality of iron, or the losses of energy in iron due to magnetic reversals, none have been successful as accurate means of measurement that have not given simultaneous and independent readings (capable of interpretation in absolute units) of the two quantities, magnetic induction or intensity of magnetization, and the magnetizing force producing that induction.

In a magnetic-curve tracer intended for accurate work, the first condition to be fulfilled is that its indicating system shall be capable of receiving and recording two simultaneous and independent displacements, always at right angles to each other, and always strictly proportional, respectively, to the intensity of magnetization of the sample under test, and the magnetizing force producing that magnetization.

\footnotetext{
* Communicated by the Author.
} 
This condition is not fulfilled by any magnetic-curve tracer, so far as I know, that has ever been described. The failure being due to mutual interference (mechanically or magnetically) of the separate displacements of the indicating system, to lack of proportionality of these two displacements to the intensity of magnetization and magnetizing force respectively, or to the inability to interpret these displacements in terms of Absolute Units.

In the following description of a continuous and alternating current magnetic-curve tracer, the order observed will be the actual order of development from the observation of the underlying principle to the embodiment of this principle in the present form of instrument.

$\$ 2$. Several years ago (in the early part of 1892 ), while making an investigation of the losses in iron magnetized by alternating currents, the writer used, he believes for the first time, an instrument which may be conveniently called an alternating-current magnetometer. A small helix of fine insulated wire was suspended bifilarly, so that the axis of the helix was in the earth's magnetic meridian. The lower ends of the bifilar wires were put in connexion with the free ends of the helix, and the upper ends of the bifilar wires were arranged so that the suspended helix might be made to form part of a circuit, composed of a few cells of secondary battery, a regulating resistanee, and a revolving contact-maker carried on the shaft of an alternating-current generator. It is clear that when the armature of the alternating-current generator was set in rotation at a constant angular velocity, an intermittent current of constant mean intensity and of definite period and phase relation was passed through the suspended helix. It was observed, on bringing a long rod magnetized by an alternating current from the generator whose armature shaft carried the contact-maker, that the suspended helix was deflected from its position of rest through a definite angle for any definite position of the rod; and that for any fixed position of the rod, by varying the point in the revolution of the armature-shaft at which the intermittent current was made through the suspended helix, a series of deflexions of the suspended helix to the right and left of the zero position was obtained. Thus, by means of the suspended helix carrying an intermittent current of proper periodic time and of constant mean intensity wo are enabled to measure directly the magnetic state of the iron at any definite point of the alternating-current waves. The alternating-current magnetometer method, employed to measure the losses in iron magnetized by alternating currents, has been described in the 
London 'Electrician' of Feb. 3, 1893, and also in the New York 'Electrical World' of Feb. 4, 1893, and need not be entered into here.

Although the alternating-current magnetometer can plainly be used equally well for continuous and alternating currents of any frequency, yet as a method of measuring losses in iron under widely varying conditions of magnetization and periodicity it necessitates the separate observation of a comparatively large number of quantities, the subsequent calibration of these quantities, and finally the graphical representation of them, before the desired estimation of losses can be made.

$\$ 3$. It seemed desirable, therefore, in order to facilitate the investigation of the nature and amount of the losses in iron, carried through magnetic cycles varying widely in both amplitude and in time, to devise an instrument that would, first, be independent in its action of the time required to complete the magnetic cycles; second, be accurate and reliable, and give indications capable of interpretation in Absolute Units ; third, that would reduce the number of observations required to obtain the losses to an absolute minimum; fourth, that would read directly the quantities to be measured; and fifth, that would allow measurements to be made in the least possible time.

It is attempted to fulfil these conditions in the present instrument in the following way:-A system of two small helices is so suspended and mechanically connected that any angular displacement about a vertical axis, given the one, is imparted to the other also, while one of the two helices may receive independent angular displacements about a horizontal axis besides. The arrangement and connexions of the magnetometer system will be immediately seen from the accompanying diagram (fig. 1). If the losses in iron magnetized by an alternating current are to be measured, an intermittent current of the proper periodic time (secured, as previously indicated, by a revolving contact-maker on the shaft of the alternating-current generator) is passed through the helices of the suspended magnetometer system in series, making of the small helices during the very short time the current is flowing in them virtual magnets. If now the sample to be tested, in the form of a long rod supplied with the proper magnetizing coil, be adjusted so that its axis is vertical and lies in the vertical axis of suspension of the magnetometer system, and it be magnetized by an alternating current from the generator whose armature-shaft carries the revolving contact-maker, only that one of the magnetometer helices which is capable of displacement about a horizontal 
axis (called hereafter the I-magnetometer helix) will be deflected. The horizontal suspension being a silver unifilar

Fig. 1.-Magnetometer System.
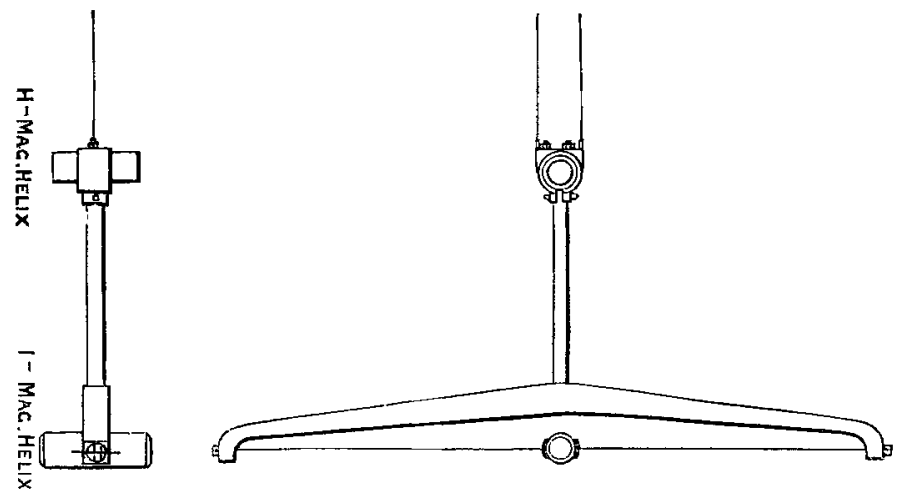

torsion-wire, the angular displacements of the helix may be taken proportional in magnitude to the product of the magnetic moments of the long rod and the helix, at the point in the alternating current waves at which the helix becomes a magnet. As will be seen on referring to eurve (a), fig. 2,

Fig. 2.-Calibration of Magnetic Curve Tracer.

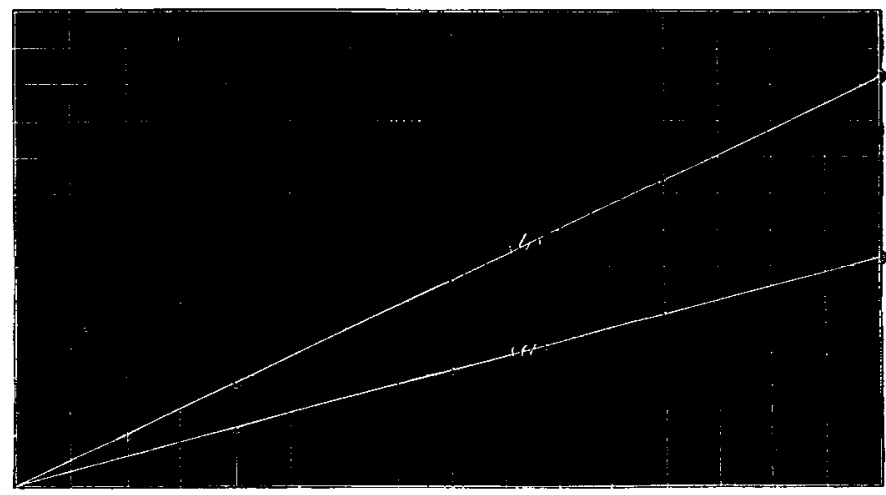

(a) Ordinates $=$ deflexion of I-magnetometer helix.

Absciseæ = magnetic force at centre of I-magnetometer helix.

(b) Ordinates = deflexion of $\mathrm{H}$-magnetometer helix.

Abscissæ $=$ current in $\mathrm{H}$-deflecting coils.

by keeping the mean strength of the intermittent current constant throughout an experiment, the vertical displacements of the I-magnetometer helix may be taken proportional to the 
magnetic moments of the sample, at the points in the alternating-current waves at which the instantaneous direct current passes in the magnetometer system. Since the centre of the I-magnetometer helix lies in the prolongation of the axis of the long rod, and the magnetic field due to the long rod is symmetrical about its axis, the deflecting force exerted on the I-magnetometer helix by the long rod is independent of the angular position of the I-magnetometer helix about the vertical axis of suspension of the magnetometer system. If, then, we adjust a coil of wire so that its centre is in a horizontal line (normal to its plane) passing through the centre of, and perpendicular to that magnetometer helix capable of displacement about a vertical axis only (called hereafter the H-magnetometer helix), and pass through this coil the same alternating current that magnetizes the sample, the $\mathrm{H}$-coil will receive a horizontal angular displacement, and through the mechanical connexions of the parts of the moveable system it will carry with it through the same horizontal angle the I-magnetometer helix. This hori\%ontal displacement being made against the restoring couple of the fine silver wire bifilar suspension of the system, is equilibrated partly by the restoring couple of the bifilar suspension proper (which varies as the sine of the angle of displacement), and partly by the torsion of the bifilar wires (which varies directly as the angle of displacement). Hence for small horizontal angular deflexions the angular displacement may be taken proportional to the product of the magnetic moments of the coil carrying the magnetizing current and the H-magnetometer helix, at the point in the alternating current waves at which the helix becomes a magnet. But the magnetic moment of the H-magnetometer helix may be kept constant throughout an experiment; hence, as will be seen from curve (b), fig. 2, the horizontal angular displacements may be taken proportional to the magnetic action, or the magnetizing force of the current, at the points in the alternating-current wayes at which the instantaneous direct current passes through the magnetometer helices.

If it is desired to measure the losses of energy in iron magnetized by continuous currents, or the "static" losses of iron, plainly the only change of arrangement in the curvetracer is to substitute for the intermittent current through the magnetometer system a direct current of the same mean intensity, to replace the source of alternating current by a continuous-current source, and provide a regulating resistance for varying the continuous magnetizing current from zero to the maximum value required. 
In either case, by means of one of the mirrors attached to the I-magnetometer helix, we are enabled to record a displacement, the rectangular components to which are independent of each other, and accurately proportional to the intensity of magnetization of the sample and the magnetizing force producing that magnetization, at any point in the magnetic cycle.

Thus we have, in this magnetometer system of two simple belices connected as described, the fulfilment of all the essential conditions for an instrument measuring the dissipation of energy in either "static" magnetic cycles, or in "periodic" magnetic cycles of any frequency.

$\$ 4$. Fig. 3 shows the form of the magnetic-curve tracer Fig. 3.

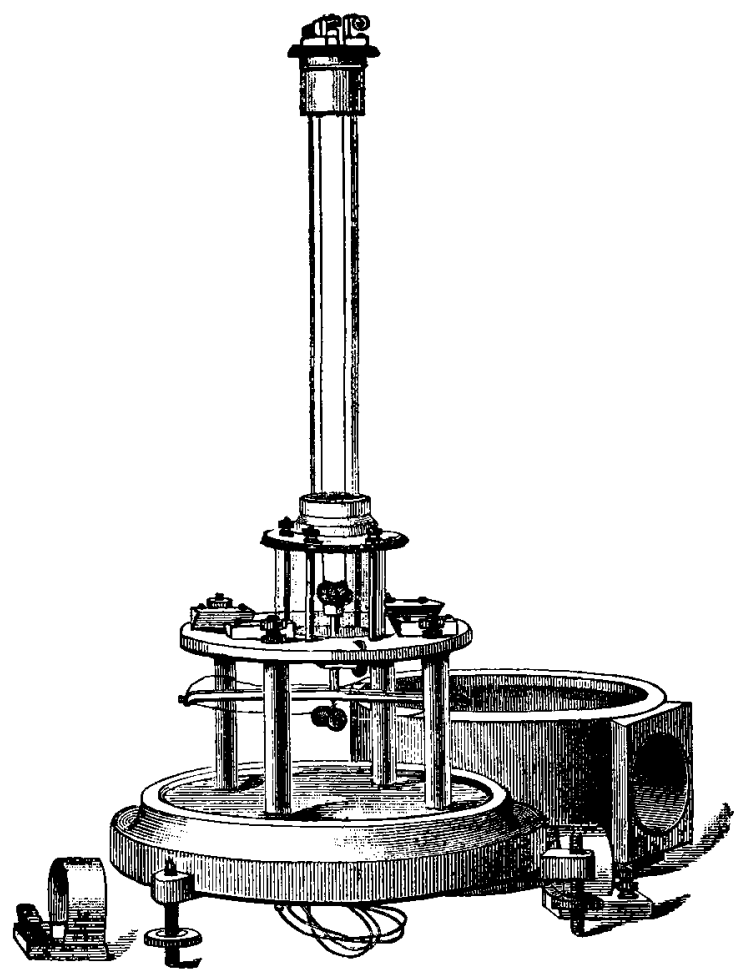

now used in the Magnetic Observatory of Princeton University. It consists, first, of a solid wooden base of circular shape, provided with levelling-screws. From this base arise four vulcanite pillars, which support a vulcanite disk, having 
a circular opening at its centre. The cover of the instrument rests in a groove in the wooden base, fitting closely about the circular disk at its top, and is provided with a plane glass window for the admission of a beam of light. Placed diametrically opposite, on the upper surface of the vulcanite disk, are two coils of a few turns of stout wire, hereafter to be called the H-deflecting coils. From the centre of this same disk rises a glass cylinder, covered at its upper end by a second vulcanite disk, to which is attached the suspensiontube. The suspension-tube carries at its upper end a torsion head, provided with binding-posts and adjusting devices for regulating the bifilar wires. From this torsion-head is suspended, by means of the silver bifilar wires, the moveable magnetometer system. The upper or H-magnetometer helix is held by a hard rubber clamp, serving at the same time as a terminal block for the lower ends of the bifilar wires. To the lower part of this clamp is fastened a hard rubber shaft, supporting centrally, at its lower end, a horizontal hard rubber bar, to the extremities of which are fastened (by means of suitable adjusting devices) the horizontal unifilar torsion-wires carrying the I-magnetometer helix.

Each of the magnetometer helices having the same number of turns of wire will, when so connected as to develop opposite polarities in the ends lying in the same direction, form an astatic combination for horizontal deflexions. If it should be necessary to make the vertical deflexions also independent of the earth's magnetic field, one can easily arrange a flat coil of wire with its centre in the vertical axis of suspension of the moveable system, and its plane horizontal, and pass through the coil such an electric current as will just equal and neutralize, in its magnetic effect, the vertical component of the earth's magnetic field at the centre of the I-magnetometer helix.

$\S 5$. In using the instrument, it should be set up on some firm support (generally, though not necessarily, so that the planes of the H-deflecting coils are parallel to the earth's magnetic meridian) and properly levelled until the suspended system is perfectly free, and hangs centrally in the instrument. The length of the bifilar wires should then be adjusted until the centre of the H-magnotometer helix lies in a horizontal line passing through the centre of the $\mathrm{H}$-deflecting coils on either side of the instrument. The stress on the bifilar wires should then be equalized, by means of the adjusting devices at either end of the horizontal bar carrying the I-magnetometer helix, the horizontal torsion-wires should be regulated in length, so that the centre of gravity of the 
I-magnetometer helix lies in the vertical axis of the suspended system, and the torsion adjusted in the wires until the axis of the helix is horizontal. 'The torsion-head of the instrument is then turned until the axes of the magnetometer helices are parallel to the planes of the $\mathrm{H}$-deflecting coils.

Upon one of the mirrors, carried by the I-magnetometer helix, is projected a beam of light from some fixed source, which after reflection is received upon a screen placed normally to the helices in their undisturbed position. By means of crosshairs placed in the path of the incident beam of light, one is enabled to mark on the screen the initial or zero position of the magnetometer system, and to measure any subsequent angular displacement of either or both of the magnetometer helices.

The sample to be tested (in the form of a long rod supplied with the proper magnetizing coil) should be placed with its axis in the vertical line of suspension of the magnetometer system. This can be accomplished most conveniently by clamping the sample and its magnetizing coil below the support of the instrument. The sample and its magnetizing coil should then be adjusted vertically, until the magnetic action of the sample, for maximum intensity of magnetization, produces the desired maximum vertical deflexion of the Imagnetometer helix. A compensating coil of a few turns of wire should then be adjusted so as to neutralize, at the 1magnetometer helix, the magnetic action of the magnetizing coil about the sumple.

The accuracy of the adjustments for horizontal displacements can be tested by making the magnetometer helices active, by means of a constant current of one or two tenths of an ampere, and reversing an independent direct current through the H-deflecting coils. This should give the magnetometer system equal horizontal displacements on either side of the zero position, and, for various current-strengths, the magnitude of these deflexions should be proportional to the respective currents.

The accuracy of the adjustments for vertical displacements depends upon the setting of the sample to be tested (with its magnetizing coil) and the balancing coil. This can be tested before beginning an experiment by placing the magnetizing coil (with the sample removed) in the position it will occupy during an experiment, and reversing continuous currents of various strengths through it. There should be no horizontal displacements, and the vertical displacements, on either side of the zero position, should be proportional to the respective magnetic forces due to the magnetizing coil, at the I-magPhil. Mag. S. 5. Vol. 41. No. 249. Feb. 1896. 
netometer helix. The compensating coil should be so connected in series with the magnetizing coil as to give an opposing magnetic effect at the I-magnetometer helix, and by keeping its plane horizontal, and its centre in the vertical line of suspension of the magnetometer system, be adjusted in position until the joint magnetic effect of the two coils at the I-magnetometer helix is zero, for all strengths of current through them.

$\S 6$. The instrument, having thus heen set up and adjusted and the adjustments tested, is ready to be used for the measurement of energy-losses in iron magnetized by either direct or alternating currents. If the losses in iron magnetized by continuous currents are to be measured, a current of two-tenths of an ampere from some independent battery (a single cell of storage-battery answers very well) is passed through the helices of the magnetometer system, and kept constant throughout an experiment. The intersection of the cross-hairs in the beam of light reflected upon the recording screen by one of the mirrors carried by the I-magnetometer helix, is taken as the centre of a system of vertical-horizontal rectangular coordinates. The sample is then placed in the magnetizing coil, which, after being properly connected in series with the compensating coil, the H-deflecting coils of the instrument, a regulating resistance, and a source of continuous current, has the magnetizing current through it increased from zero to the maximum value required to produce the desired maximum degree of magnetization, reduced to zero, reversed, the same operation performed in the opposite sense, and the cycle completed by returning finally to the first maximum. The point of intersection of the cross-hairs on the screen will be displaced for every different value of the magnetizing current, a distance from each of the coordinate axes previously drawn, proportional respectively to the magnetizing force acting on the sample and the intensity of magnetization of the sample. Hence by marking down on the screen the point of intersection of the cross hairs in the reflected benm of light, for any desired number of values of the magnetizing current thronghout the cycle of magnetization, we have an accurate outline of the magnetization curve for the sample, or, as it is frequently called, the "hysteresis" curve. By properly varying the magnetizing current, loops can be traced to any part of the hysteresis curre, or a complete set of graded cycles obtained without removing the sample.

In measuring the losses in iron magnetized by an alternating current, an intermittent current of a mean value of 
two-tenths of an ampero (obtained by means of a revolving contact-maker on the shaft of the alternating-current generator supplying the alternating magnetizing current) is passed through the magnetometer helices, and kept accurately constant throughout an entire experiment. For the continuous magnetizing current is substituted an alternating current, adjusted to such a mean value as will give the desired maximum intensity of magnetization. As has been shown, by varying the point in the revolution of the armature-shaft at which contact is made, or current passes in the magnetometer helices, different deflexions will be obtained, the rectangular components to which are strictly proportional to the magnetizing force, and the intensity of magnetization respectively, at the points in the alternating-current waves at which the instantaneous direct current passes through the magnetometer helices. Hence by varying the point of contact on the revolving contact-maker, through a circular angle corresponding to 360 degrees of phase in the alternatingcurrent waves, we have, as in the case of "static" cycles, the successive positions on the screen of the intersection of the cross-hairs in the reflected beam of light, marking points in the outline of the energy-loss curve.

\$7. In calibrating the instrument for either "static" or "periodic" cycles, the current through the magnetometer helices is kept the same in kind and magnitude as during the experiment. The sample is removed from the magnetizing coil, and an accurately measured current sent through either the magnetizing coil or the compensating coil, alone. The deflexion is marked down on the vertical axis of coordinates. Then an accurately measured current is sent through the $\mathrm{H}$ deflecting coils alone, and the resulting deflexion marked down on the horizontal axis of coordinates.

By knowing the distance of the resultant magnetic distribution, or magnetic poles, from the I-magnetometer helix, we can calculate, by well-known magnetometer laws, the value of the magnetic force at the centre of the I-magnetometer helix, due to the long rod, in terms of the distance of the poles from the centre of the helix, the cross section of the sample, and the intensity of magnetization of the sample. It will be plain, by considering the relative position of the long rod and l-magnetometer coil, that this force,

$$
\mathrm{F}_{l r}=\mathrm{SI}\left(\frac{1}{\mathrm{R}^{2}}-\frac{1}{\mathrm{R}^{\prime 2}}\right) ; \quad \cdot \quad \cdot \quad \cdot \quad .
$$

where $\mathrm{S}$ is the cross section of the sample, I the intensity of magnetization of the simple, $R$ the distance from the 
centre of the I-magnetometer helix to the nearer pole, and $R^{\prime}$ the distance from the centre of the I-magnetometer helix to the farther pole.

By knowing the distance of the compensating coil during calibration from the I-magnetometer helix, its number of turns, mean radius, and the current through it, we are enabled to calculate its magnetic force at the I-magnetometer helix. If the calibrating coil is a flat coil of only a few turns of wire (as is always most convenient), then, as is well known, its magnetic force at the I-magnetometer helix is expressed by :

$$
\mathrm{F}_{(\mathrm{oc})}=\frac{2 \pi n \mathrm{C} r^{2}}{10\left(d^{2}+r^{2}\right)^{\frac{1}{2}}} ; \text {. . . . . }
$$

where $n$ is the number of turns in the calibrating coil, $\mathrm{C}$ the value in absolute units of the current in it, $r$ its mean radius, and $d$ the distance of the calibrating coil from the I-magnetometer helix. Calling $D_{c}$ any particular vertical deflexion in the course of an experiment, and $D_{c}$ the vertical deflexion during calibration, we have, since the current in the magnetometer system has been kept constant through the whole experiment, the following relation :-

$$
\frac{\mathrm{D}_{s}}{\mathrm{D}_{c}}=\frac{\mathrm{F}_{l_{r}}}{\mathrm{~F}_{c c}} \cdot \ldots . . . \quad . \quad . \quad .
$$

Substituting in equation (3) from equations (1) and (2) we get

$$
\frac{\mathrm{D}_{c}}{\mathrm{D}_{c}}=\frac{\mathrm{SI}\left(\frac{1}{\mathrm{R}^{2}}-\frac{1}{\mathrm{R}^{\prime 2}}\right)}{\frac{2 \pi n\left(r^{2}\right.}{10\left(d^{2}+r^{2}\right)^{\frac{3}{2}}}}
$$

Solving equation (4) for I, we get

$$
\mathrm{I}=\frac{2 \pi n \mathrm{Cr}^{2}}{10\left(d^{2}+r^{2}\right)^{\frac{3}{2}}} \frac{\mathrm{R}^{2} \mathrm{R}^{\prime 2}}{\mathrm{SR}^{\prime 2}-\mathrm{R}^{2}} \frac{\mathrm{D}_{e}}{\mathrm{D}_{e}}
$$

We are thus enabled to find the value of the intensity of magnetization of the sample in Absolute Units for any point on the entire curve of magnetization.

Knowing the number of turns, the length, and the current flowing in the magnetizing coil surrounding the sample, we can at once calculate in Absolute Units the magnetizing force of the coil on the sample. In the calibration of the horizontal deflexions, we know the current flowing in the $\mathrm{H}$ deflecting coils and the displacement it gives along the horizontal axis. We calculate the magnetizing force of the 
magnetizing coil about the sample for that current, and by direct proportion betweon horizontal displacement in calibration, and the horizontal displacement of any point on the curve, we obtain in Absolute Units the magnetic force corresponding to any point on the curve. Having thus calibrated the curves of either continuous or alternating current magnetic cycles in Absolute Units, the energy losses, $\int I d \mathrm{H}$, may be obtained by taking the area of the curves in the usual way.

Although this description has only been concerned with the curve-tracer as a means of measuring the magnetie quality of, and the energy losses in iron and other metals, when carried through magnetic cycles, it plainly lends itself to such operations as direct tracing of alternating-current and electromotive-force curves, the investigation of the nature and amount of iron losses in alternating-current transformers, the measurement of the power in any electrical circuit; \&c.

XIV. On the Dissociation Degree of some Electrolytes at $0^{\circ}$. By R.W. Wood*.

TYHE values obtained by the lowering of the freezing-point 1 for the dissociation-degree of dissolved electrolytes are always a little smaller than those calculated from the electrical conductivity.

Meyer Wildermann $\dagger$ has recently lessened the difference by the use of a more accurate method for the freezing-point determinations, and has expressed the opinion that the cause of the discrepancies lay in the fact that the electrical conductivities have been determined at a higher temperature $\left(18^{\circ}-25^{\circ}\right)$.

At Prof. Jahn's suggestion, I have determined the conductivity of certain Salts and Acids in solutions of varying concentration at $0^{\circ}$, for the purpose of reckoning the Dissociation-degree at this temperature, and the results indicate that, in dilute solutions, the dissociation-degree is practically independent of the temperature.

The determinations were made according to the Kohlrausch method, and the conductivity of the distilled water used in the experiments was determined at different temperatures and taken into account.

The value expressing the conductivity of. infinitely dilute

* Translated from the Zeitschrift für phys. Chemie, xviii. p. 3 (1895). Communicated by the Author.

$\dagger$ Phil. Mag. July 1895. 